\title{
Mesenchymal stem cells modifications for enhanced bone targeting and bone regeneration
}

\author{
Yuliya Safarova1,2, Bauyrzhan Umbayev1, Gonzalo Hortelano³ \& Sholpan Askarova*,1(iD) \\ ${ }^{1}$ Center for Life Sciences, National Laboratory Astana, Nazarbayev University, Nur-Sultan, Kazakhstan \\ ${ }^{2}$ School of Engineering \& Digital Sciences, Nazarbayev University, Nur-Sultan, Kazakhstan \\ ${ }^{3}$ School of Sciences \& Humanities, Nazarbayev University, Nur-Sultan, Kazakhstan \\ *Author for correspondence: Tel.: +7 717270 6514; +7 777772 7828; shaskarova@nu.edu.kz
}

In pathological bone conditions (e.g., osteoporotic fractures or critical size bone defects), increasing the pool of osteoblast progenitor cells is a promising therapeutic approach to facilitate bone healing. Since mesenchymal stem cells (MSCs) give rise to the osteogenic lineage, a number of clinical trials investigated the potential of MSCs transplantation for bone regeneration. However, the engraftment of transplanted cells is often hindered by insufficient oxygen and nutrients supply and the tendency of MSCs to home to different sites of the body. In this review, we discuss various approaches of MSCs transplantation for bone regeneration including scaffold and hydrogel constructs, genetic modifications and surface engineering of the cell membrane aimed to improve homing and increase cell viability, proliferation and differentiation.

First draft submitted: 2 July 2019; Accepted for publication: 30 March 2020; Published online: 16 April 2020

Keywords: bone regeneration $\bullet$ cell surface engineering $\bullet$ genetic modifications $\bullet$ hydrogels $\bullet$ MSCs $\bullet$ scaffolds

A bone fracture is a common medical condition, which can be due to traumatic injury or as a result of pathological weakening of the bones. The severity of the injury depends on the fracture (type, magnitude and location) and increases with age [1]. Some fractures require only temporary fixation and protection, while other serious fractures (in elderly, critical size bone defects, bone tumor surgery, pathologic fractures etc.) undergo a more difficult process of natural regeneration and often fail to heal. These conditions are called a 'nonunion' and a 'delayed union' of fractures.

Osteoporosis is the most common systemic bone disorder that predisposes the affected individuals to pathological bone fractures. Furthermore, fractures in elderly osteoporotic patients are challenging to treat due to prolonged healing time and the complexity of surgical fracture fixation in a weakened bone [2]. Second, the most common pathological bone condition is Paget's disease of bone [3]. The etiology of the Paget's disease of bone is still unknown and may include genetic factors as well as environmental. In this condition, the normal bone equilibrium is shifted toward the bone resorption processes which inevitably alters the fracture healing process [4]. Both disorders are primarily diagnosed in older people and in rare cases in people less than 55 years old [3,5]. Besides the aging-associated pathological conditions, there is also a group of genetically induced bone disorders. The genetic condition known as osteogenesis imperfecta is characterized by a defect in the collagen production genes (COL1A1 or COL1A2 gene), which results in bone malformation and impairment in the bone regeneration process [6]. Some other conditions are also able to worsen bone regeneration. For example, bacterial osteomyelitis impairs bone remodeling and leads to uncontrolled bone loss [7]. There are also a group of auto-inflammatory bone diseases that can affect proper bone healing including chronic nonbacterial osteomyelitis, Majeed syndrome, deficiency of IL-1 receptor antagonist and cherubism [8].

Many innovative therapeutic strategies have been identified in recent years to improve bone regeneration, and mesenchymal stem cell (MSCs) transplantation is one of the promising approaches. Overall the properties of MSCs make them very suitable biological material for transplantation in bone-associated conditions and fractures. MSCs are able to differentiate into the target tissue; they have advantageous immune modulating properties and 
provide growth factors to facilitate the repair process. The stimulation of the natural repairing processes by providing osteoblasts precursors (MSCs) that will home and attach firmly to the site of injury increases the rate of regeneration of bone structures and improve healing. Transplantation of MSCs into the injured area can promote healing not only by directly increasing the number of precursor cells but also through a paracrine effect by releasing growth factors and immunomodulatory cytokines and chemokines to induce regeneration [9].

MSCs have been isolated so far from various types of tissues, including bone marrow, adipose tissue, synovial membrane and fluid, human placenta, umbilical cord, amniotic fluid or various fetal tissues. Although MSCs from these different sources have been applied in preclinical models, bone marrow-derived MSCs (BM-MSCs) and adipose-derived MSCs (AD-MSCs) are the most commonly used cell types for bone regeneration [10,11]. BM-MSCs are isolated at a relatively low density and must be expanded in vitro, in contrast with AD-MSCs that can be harvested in higher quantities [12]. However, BM-MSCs show more expression of osteogenic differentiation genes [11], while AD-MSCs show stronger angiogenic potential [13], and is a promising tool in the treatment of vascular ischemic conditions [14].

There are two main routes of transplantation of MSCs: systemic and local. Systemic administration involves intravenous (IV) and intra-arterial (IA) injection of the cells, while local administration involves direct injection of the cells into the regeneration site. Systemic route is often less invasive and keeps the cells close to the source of oxygen and nutrients with the ability to extravasate into the target tissue [15]. However, previous research in this area showed that, upon systemic administration, most of the transplanted MSCs are concentrated in the lungs [16-19], though after 10 days, the percentage drops dramatically - to $2 \%$ compared with the initial $35 \%$ [20]. Migration of transplanted MSCs from the lungs is believed to be driven by inflamed organs [21]. Due to their nature, MSCs can sense chemokine CCL21 in vessels near the sites of inflammation and some MSCs escape the lungs and home to inflamed tissue [22]. Thus, the efficacy of systemic MSCs administration in cases of bone pathology could be affected by other underlying chronic conditions. Another disadvantage of the systemic MSCs administration is the aggregation of the transplanted cells in the areas of abnormal cancerous cell proliferations such as breast or ovary cancer [23].

There are several ways to target MSCs to the tissue of interest. The targeting moiety can be induced by an independently administrated component, for example, an injection of parathyroid hormone (PTH). It has been shown that therapy with PTH together with MSCs transplantation increases cell migration to the site of the bone defects and improves further differentiation of the cells [24]. In general, recruitment of the MSCs to the site of fracture is activated through the stromal cell-derived factor 1/C-X-C CXCR4 axes. However, PTH administration shifts the mechanism of MSCs recruitment to the amphiregulin pathway in which EGF-like ligand is secreted in the damaged area [24,25].

Another approach is a local transplantation of MSCs to the site of bone fracture. A major advantage of local cell delivery is the close proximity of the transplanted cells to the areas of bone defect. However, the survival of those cells is questionable since oxygen and nutrients are not always available at the sites of injection. Therefore, in order to increase the degree of cell engraftment, the delivery system must place cells at, or allow MSCs to migrate to the site of bone defect. Thus, the main focus of this review is modifications of MSCs that are aimed at improving the bone targeting potential of the MSCs and enhancing survival of cells upon therapeutic transplantations.

In this review, we refer to MSCs as a multipotent stem cells that according to International Society for Cell and Gene Treatment fulfills three main criteria: first, MSCs are adherent to plastic in culture; second, they express CD73, CD90, CD105 on their cell surface membrane and lack CD14, CD34, CD45 and human leukocyte antigen - DR isotype HLA-DR) expression; finally, MSCs can differentiate down the osteogenic, chondrogenic and adipogenic lineage [10].

\section{Scaffolds \& hydrogels}

The use of biomaterial scaffolds is one of the most widely used strategies to enhance repair processes in bone tissue. The organic and inorganic materials implemented in scaffold manufacturing, including polymeric constructs, ceramics, metals and natural matrices are summarized in the scheme below (Figure 1). A precise review of all currently available scaffold materials for bone tissue engineering was given by Ghassemi $e t$ al. [26]. The authors concluded that different approaches should be combined in order to provide the scaffold with better mechanical strength (that lack both synthetic and natural polymeric scaffolds), less fragility (than ceramics scaffolds) and the incorporation of biologically active agents in order to promote osteoinductivity. 


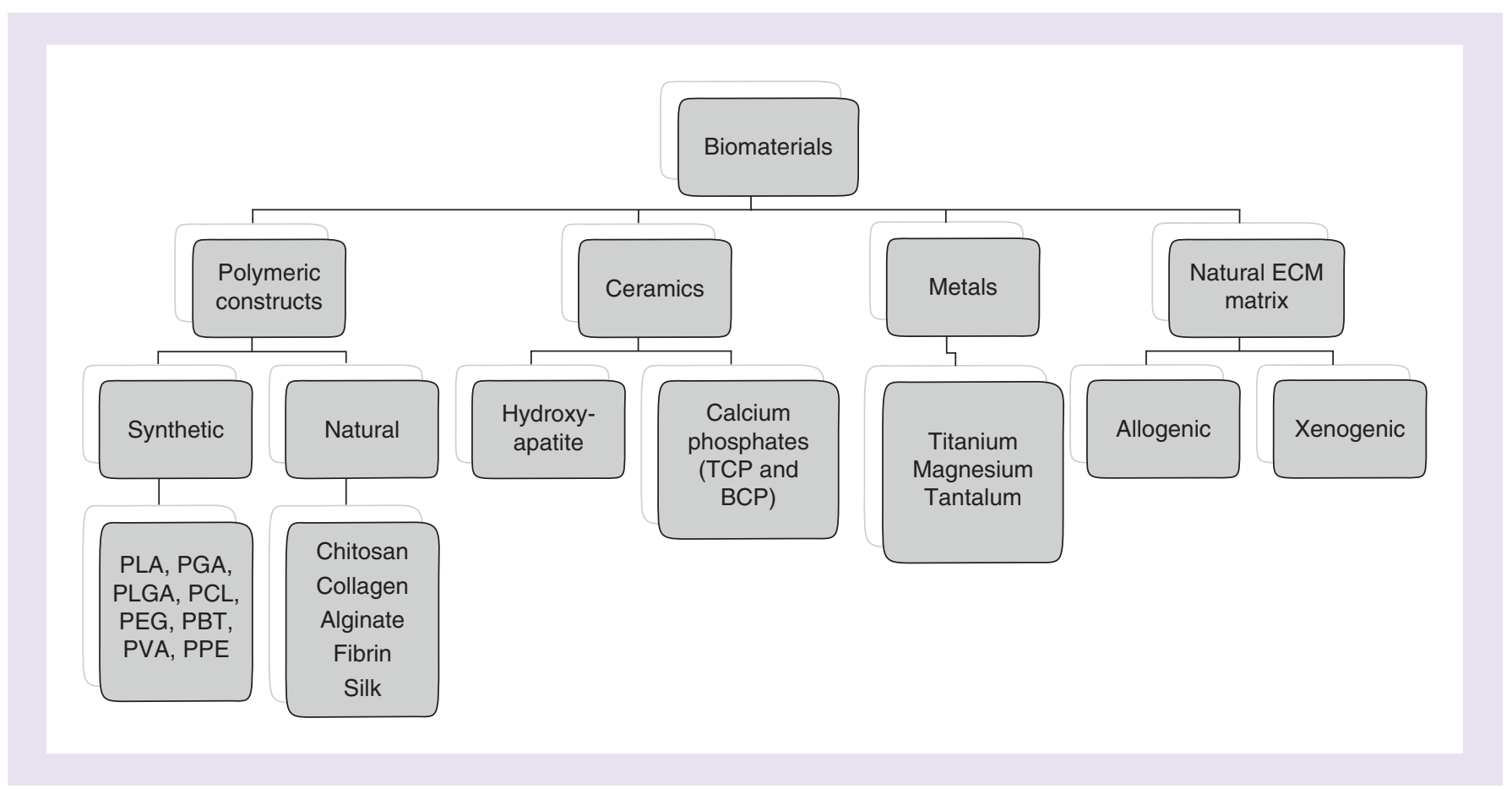

Figure 1. Materials implemented in scaffold manufacturing.

ECM: Extracellular matrix; PBT: Polybutylene terephthalate; PCL: Polycaprolactone; PEG: Polyethylene glycol; PGA: Polyglycolic acid; PLA: Polylactic acid; PLGA: Poly(lactic-co-glycolic acid); PPE: Polyphenyl ether; PVA: Polyvinyl alcohol; TCP: Tricalcium phosphate.

Seeding scaffolds with osteoblast precursor cells is a promising approach to increase the efficacy of scaffold transplants. Ceramic scaffolds are very suitable for filling critical size bone defects because the scaffold matrix provides mechanical support for the cells to proliferate, differentiate into osteoblasts and eventually calcify. In a study by Agacayak and colleagues, the combination of MSCs, platelet rich plasma and biphasic calcium phosphate construct has been demonstrated to be a more effective approach for inducing osteogenesis in rat calvarial bone defect than the use of ceramic bone scaffold alone [27]. Similarly, hydroxyapatite ceramic scaffolds seeded with culture-expanded BM-MSCs were able to regenerate critical size bone defects of tibia diaphysis in sheep to a greater extent than synthetic bone substitute alone [28]. Several clinical cases were reported on the use of $\beta$-tricalcium phosphate scaffolds to meet craniomaxillofacial applications [29-33]. A $\beta$-TCP scaffold loaded with AD-MSCs and BMP-2 was used to reconstruct a maxillary defect left after the removal of keratocyst. The construct was first implanted into a muscle for ectopic bone formation and then transplanted into the maxillary area and led to successful healing 4 month after surgery [31].

Yet, the interactions between scaffolds and MSCs involve many factors affecting stem cell survival, proliferation and differentiation. One of the very important issues in cell-scaffold constructs is the lack of oxygen supply and nutrients, since vessel formation is a slow process. In this regard, a number of recent studies have been directed toward creating bioactive scaffolds and hydrogels that promote angiogenesis while supporting cell proliferation and differentiation (Table 1) [34,35].

For example, Yu et al. seeded the polycaprolactone-hydroxyapatite scaffolds with a combination of osteoblast precursor cells and endothelial cells to enhance angiogenesis, which significantly improved the bone regeneration process [38]. Porous silk scaffolds seeded with BM-MSCs in a study carried out by Zhang et al. was successful and provided evidence for increased rate of regeneration of cranial critical size bone defects in rats [39]. The authors showed that cells were able to survive up to 8 weeks due to the presence of VEGF and BMP-2 factors that promoted angiogenesis and the subsequent differentiation of cells along the osteogenic lineage. Similarly, transplantation of MSCs entrapped into the collagen sponge/hyaluronic acid-based hydrogel complex scaffolds containing VEGF and BMP-2 resulted in a significant increase of bone mineral density at canine maxillary alveolar bone defects [40]. Zhao et al. encapsulated BMSCs and BMP-2 into photocross-linkable hydrogel microspheres composed of gelatinmethacryloyl chloride and demonstrated improved bone formation of the rabbit femoral ankle [41]. 
Table 1. Bioactive hydrogels and scaffolds for bone regeneration.

\begin{tabular}{|c|c|c|c|}
\hline Scaffold/hydrogel carrier & $\begin{array}{l}\text { Biologically active } \\
\text { agent }\end{array}$ & Outcome & Ref \\
\hline Poly(lactic-co-glycolic acid) & VEGF & Bone formation in an irradiated rat calvarial defect & [36] \\
\hline A 3D honeycomb-like PCL scaffold & rhBMP2-PCL & Promotes bone healing in a large bone defect of rabbit ulna. & [37] \\
\hline Cylindrical porous PCL-HA scaffolds & Osteoblasts and ECs & $\begin{array}{l}\text { A widely distributed capillary network, osteoid generated by } \\
\text { osteoblasts and absent ischemic necroses in a } 0.4-\mathrm{cm} \text {-long } \\
\text { segmental femur defect of BALB/C mice }\end{array}$ & [38] \\
\hline Silk scaffolds & $\begin{array}{l}\text { BMSCs, VEGF and } \\
\text { BMP-2 }\end{array}$ & Regeneration of critical size cranial bone defects in rats & [39] \\
\hline $\begin{array}{l}\text { Collagen sponge/hyaluronic acid-based hydrogel complex } \\
\text { scaffolds }\end{array}$ & VEGF and BMP-2 & $\begin{array}{l}\text { Increase of bone mineral density at canine maxillary alveolar } \\
\text { bone defects }\end{array}$ & [40] \\
\hline $\begin{array}{l}\text { Photocrosslinkable hydrogel microspheres composed of } \\
\text { gelatin-methacryloyl chloride }\end{array}$ & BMSCs and BMP-2 & Improved bone formation in rabbit femoral ankle & [41] \\
\hline Nano calcium sulfate/alginate scaffold & $\begin{array}{l}\text { BMP-2 transfected } \\
\text { MSCs }\end{array}$ & Bone bridging of critical-sizes calvarial bone defects in rats. & [42] \\
\hline Alginate microcapsules & $\begin{array}{l}\text { Fibrinogen, fibronectin } \\
\text { or RGD }\end{array}$ & $\begin{array}{l}\text { Increased viability, proliferation and osteogenic differentiation } \\
\text { of enclosed MSCs }\end{array}$ & [43-45] \\
\hline $\begin{array}{l}\text { High guluronic acid-content alginates at hydrogel with } \\
\text { elasticity of } 60 \mathrm{kPa}\end{array}$ & hMSCs & Bone formation in nude rats with cranial defects & [46] \\
\hline Hydrogel scaffolds derived from bone extracellular matrix & Dental pulp stem cells & $\begin{array}{l}\text { Cell survival, upregulated expression of RUNX-2, osteocalcin } \\
\text { and bone sialoprotein }\end{array}$ & [47] \\
\hline$\beta$-tricalcium phosphate and BMP-2 & $\begin{array}{l}\text { Adipose derived stem } \\
\text { cells }\end{array}$ & Maxillary defect healing & {$[30,31]$} \\
\hline
\end{tabular}

He et al. demonstrated that encapsulation of BMP-2-transfected MSCs into nano calcium sulfate/alginate scaffold resulted in bone bridging of critical-sizes calvarial bone defects in rats [42]. Sayyar et al. enclosed human MSCs in biomimetic microcapsules made with alginate, a natural carbohydrate from seaweed. The decoration of alginate with fibrinogen, fibronectin or RGD (the tripeptide Arg-Gly-Asp) led to a higher viability of encapsulated human MSCs [43]. Furthermore, alginate decorated with either fibrinogen or fibronectin, but not RGD increased cell proliferation and osteogenic differentiation of enclosed MSCs [44,45].

To enrich the hydrogel scaffold with oxygen available to MSCs, Kimelman-Bleich used perfluorotributylamine (PFTBA) [48]. PFTBA is a type of perfluorocarbons that traps oxygen due to high oxygen solubility, in other words, $35 \mathrm{~mm}$ compared with the $2.2 \mathrm{~mm}$ of oxygen in water. Hydrogel with PFTBA was mixed with the cells and injected in the area of ectopic bone formation. Results showed a 2.5-fold increase in bone formation compared with the hydrogel without PFTBA as well as increase in cell survival and in osteocalcin expression [48]. Study of synthetic oxygen carriers by Benjamin et al. has also led to the conclusion that PFTBA promotes cell survival especially if MSCs are encapsulated. Availability of oxygen to MSCs significantly downregulated hypoxia-related genes as well as promoted osteogenic over chondrogenic differentiation [49].

Hydrogel scaffolds could also be derived from decellularization of the extracellular matrix (ECM). ECM scaffolds hold a great potential as they contain proinflammatory cytokines, BMPs and various growth factors including VEGF [50]. Besides the biological properties, ECM also has structural properties that allow mechanical support of the cells. In a study of Paduano et al. decellularized bone ECM with dental pulp stem cells was examined in vivo. The construct of bone ECM showed an upregulated expression of RUNX-2, bone sialoprotein and osteocalcin compared with the cells cultured on the collagen Type I hydrogel scaffold [47].

Huebsch et al. were able to improve survival rate of human mesenchymal stem cell (hMSC) by modulating elastic modulus of the hydrogels composed of high guluronic acid-content alginates (medium viscosity high-guluronic acid alginate [MVG]; FMC BioPolymer). The authors used as model nude rats with cranial defects and demonstrated that the most prominent regenerative effect occurred using a hydrogel elasticity of $60 \mathrm{kPa}$ [46]. This finding suggests that the biophysical properties of scaffold/hydrogel carriers may also play an important role in promoting the effectiveness of stem-cell based therapies. 
Table 2. Preclinical in vivo studies of genetic modified mesenchymal stem cells for bone disease treatment

\begin{tabular}{|c|c|c|c|c|}
\hline Genetic modifications & Cell type & Cellular mechanisms & Gene transfer type & Ref. \\
\hline \multirow[t]{6}{*}{ BMP-2 } & Rat bone marrow cells & Osteoblast differentiation & Adenovirus & [59] \\
\hline & Human bone marrow MSCs & & Adenovirus & [56] \\
\hline & Murine bone marrow MSCs & & $\begin{array}{l}\text { Adenovirus-associated } \\
\text { virus }\end{array}$ & [60] \\
\hline & $\mathrm{C} 3 \mathrm{H} 10 \mathrm{~T} 1 / 2 \mathrm{MSCS}$ & & Liposome & [61] \\
\hline & Murine bone marrow MSCs & & $\begin{array}{l}\text { Adenovirus-associated } \\
\text { virus }\end{array}$ & [62] \\
\hline & $\mathrm{C} 3 \mathrm{H} 10 \mathrm{~T} 1 / 2 \mathrm{MSCS}$ & & Liposome & [63] \\
\hline \multirow[t]{2}{*}{ BMP-2 and BMP-7 } & Sheep adipose-derived MSCs & Osteoblast differentiation & Adenovirus & [64] \\
\hline & Rat adipose-derived stem cell & Osteoblast differentiation & Lentivirus & [65] \\
\hline \multirow[t]{2}{*}{ BMP-2 and VEGF } & Human periosteum-derived cells & Osteoblast differentiation & Plasmid & [66] \\
\hline & Rabbit bone marrow stromal cell & Osteoblast differentiation & Adenovirus & [67] \\
\hline BMP-6 & Porcine adipose-derived stem cells & Osteoblast differentiation & Lentivirus & [72] \\
\hline BMP-6 or BMP-2 & Porcine adipose and bone marrow MSCs & Osteoblast differentiation & Nucleofection & [73] \\
\hline BMP-6 & Porcine bone marrow MSCs & Osteoblast differentiation & Nucleofection & [74] \\
\hline BMP-6 and VEGF & Rat bone marrow MSCs & $\begin{array}{l}\text { Osteoblast differentiation and } \\
\text { vasculogenesis }\end{array}$ & $\begin{array}{l}\text { Adenovirus-associated } \\
\text { virus }\end{array}$ & [75] \\
\hline BMP-7 & New Zealand white rabbit's bone marrow MSCs & Osteoblast differentiation & Adenovirus & [76] \\
\hline \multirow[t]{3}{*}{ BMP-9 } & Human MSCs & Osteoblast differentiation, ectopic bone & Adenovirus & [77] \\
\hline & Human MSCs & Osteoblast differentiation & Adenovirus & [78] \\
\hline & Rat bone marrow stromal cells & Osteoblast differentiation & Adenovirus & [79] \\
\hline Alpha-1 Antitrypsin & Murine adipose tissue-derived MSCs & $\begin{array}{l}\text { Inhibition of osteoclast-associated bone } \\
\text { resorption }\end{array}$ & Lentivirus & [86] \\
\hline Sox-11 & Rat bone marrow MSCs & MSCs differentiation and homing & Lentivirus & [87] \\
\hline Runx2 & Rat bone marrow MSC spheroids & Osteoblast differentiation & Plasmid & [88] \\
\hline Jarid1a knockdown & Rat bone marrow MSCs & $\begin{array}{l}\text { Activation of Runx } 2 \text { and subsequent } \\
\text { osteoblast differentiation }\end{array}$ & $\begin{array}{l}\text { Transfection of } \\
\text { small-interfering RNAs }\end{array}$ & [89] \\
\hline miR-135 & Rat adipose-derived MSCs & Osteoblast differentiation & Lentivirus & [90] \\
\hline miR-31 & Rat adipose-derived MSCs & Osteoblast differentiation & Lentivirus & [91] \\
\hline
\end{tabular}

\section{Genetic modifications of MSCs}

MSCs can be genetically modified to enhance their survival rate, homing efficiency and differentiation potential [5154]. Since the late $90 \mathrm{~s}$, many researches have been actively conducted to develop strategies of ex vivo gene therapy for bone engineering $[10,51,52,54-58]$. The strategies of ex vivo gene therapy are based on three pillars: the cell type, molecular target and gene transfer type (viral or nonviral; Table 2 \& Figure 2). As mentioned before, MSCs from different tissue sources have been applied in preclinical models, yet BM-MSCs and AD-MSCs are the most commonly used cell types for bone regeneration (Table 2). 


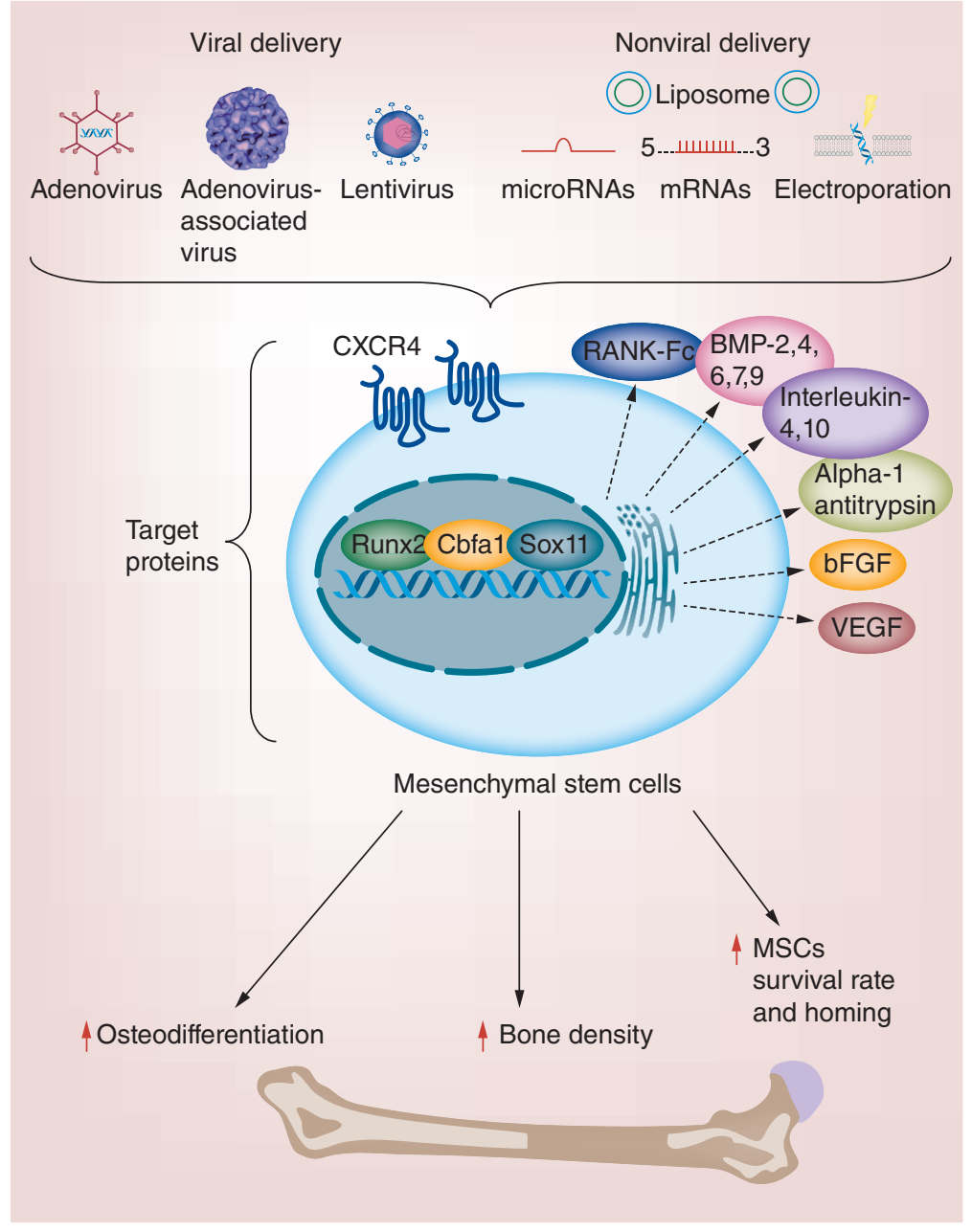

Figure 2. Viral and nonviral genetic modifications of mesenchymal stem cells for bone regeneration and treatment of bone diseases. A targeting strategy for genetic modifications of MSCs is mainly focused on three aspects: (A) activation of cytokines' secretion (BMPs, IL-4, IL-10, bFGF, VEGF) to promote MSC survival and osteogenic differentiation; (B) increasing expression of the cell surface receptors responsible for cell homing (CXCR4) or inhibition of osteoclast differentiation and activation (RANK-Fc); (C) activation of transcription factors and other proteins responsible for differentiation and migration of MSCs (Runx2, Cbfa1, Sox11). MSC: Mesenchymal stem cell; RANK-Fc: Recombinant RANK-L antagonist synthesized based on fusing the extracellular domain of RANK to the Fc portion of human immunoglobulin G(1); Runx: Runt-related transcription factor 2; Sox11: Sry-related high-mobility group box 11.

Molecular targeting strategies for genetic modifications of MSCs are mainly focused on three aspects: activation of cytokines' secretion (e.g., BMP-2, BMP-6, BMP-7, IL-4 and IL-10) in order to to promote MSC differentiation; increasing expression of the cell surface receptors responsible for cell rolling and eventual migration to the targeted tissue (e.g., CXCR4); and activation of transcription factors and other proteins responsible for differentiation and homing of MSCs (e.g., Sry-related high-mobility group box 11 [Sox11] and runt-related transcription factor 2 [Runx2]; Table $2 \&$ Figure 2).

Bone morphogenetic proteins are the most important molecular targets for bone-targeted genetic engineering of MSCs [92]. BMPs are the multifunctional cytokines belonging to a TGF $\beta$ superfamily [93]. Kang et al. demonstrated that BMP-2, BMP-4, BMP-6, BMP-7 and BMP-9 possess the highest orthotopic bone-forming activity among 14 types of human BMPs [94], and recombinant human BMP-2 and BMP-7 were approved by US FDA for treating open tibia shaft fractures and long bone nonunions, respectively [95].

Numerous investigations, pioneered by the studies of Lieberman's [59], Huard's [96] and Gazit's [56], applied BMP-2 for ex vivo gene therapy. In various experimental models, the treatment of bone defects with BM-MSCs, AD-MSCs and $\mathrm{C} 3 \mathrm{H} 10 \mathrm{~T} 1 / 2$ cells overexpressing BMP-2 led to increased bone regeneration [56-63]. A recent study has also demonstrated that BMP-2 transduced adipose-derived stem cells had higher osteogenic potential compared with BM-MSCs in vitro [97].

BMP- 4 is a key player in development of axial and craniofacial structures of the skeleton and in the early phase of fracture healing [98]. Lin et al. reported new bone formation in athymic mice transplanted with BMP-4-modified adipose-derived stromal cells from Sprague-Dawley rats [69]. In another study, the transplantation of unfractioned BM-MSCs transfected with BMP-4 improved healing of a critical-sized femur defect in adult rats [70]. Rose et 
al. have reported that BMP-4-engineered muscle-derived cells demonstrated superior results when compared with BM-MSCs in terms of improving healing of the segmental defects [99].

BMP-6 and BMP-7 are the cytokines that also possess high osteoinductive properties. For example, injection of adipose-derived stem cells overexpressing BMP- 6 gene was capable of repairing the vertebral bone void in nude rats [72]. In another study, implantation of BMP-6-modified stem cells accelerated bone regeneration of the lumbar vertebral body in the mini pig animal model [74]. Furthermore, there is data demonstrating that AD-MSCs and BM-MSCs transfected with rhBMP-6 had higher osteogenic differentiation potential compared with transfection with rhBMP-2 in vitro and in vivo [73]. In turn, BMP-7 demonstrated great therapeutic potential in the treatment of fractures resistant to healing [100]. It was reported that New Zealand white rabbit's BM-MSCs with high expression of BMP-7 combined with an nano-hydroxyapatite/collagen (NHAC) scaffold effectively repaired a rabbit radius defect [76].

A BMP-9 probably is the most effective osteoinductive growth factor among BMPs family [94]. A number of studies have demonstrated that ex vivo modification of hMSCs with BMP-9 activated endochondral bone formation [77] and promoted spinal fusion [78] in rodents. Wang et al. have revealed that BM-MSCs transfected with BMP-9 induces callus formation in rats with osteoporotic fracture [79]. Recently, it was reported that functional Notch signaling in MSCs plays a role in osteogenesis induced by BMP-9 [101].

In addition to BMPs, some other growth factors may be implicated in osteogenesis and bone regeneration. As an example, Zhang and coauthors demonstrated that MSCs, genetically modified to express bFGF, improved bone fracture healing and enhanced bone strength in mice by stimulating endogenous angiogenesis, osteogenesis and rapid cartilage turnover through endochondral ossification [82].

It is worth mentioning that bone formation is a complex process that requires fine-tuning of many signaling pathways and for optimal bone regeneration the effects of several proteins are desired [102]. In this regard, a number of studies have revealed that the use of the BMPs is the most efficient for bone formation when combined with VEGF. For example, formation of ectopic bone was more prominent after implantation of human periosteum-derived cells cotransfected with BMP-2 and VEGF [66]. Similarly, regeneration of critical-sized bone defects in rabbits was more successful when treated with a coral scafold loaded with BMP2- and VEGF-expressing BM-MSCs compared with any single factor [67]. BM-MSCs co-expressing VEGF and BMP-6 injected to avascular necrosis of the femoral head in nude mice led to the enhancement of blood vessels and bone formation [75]. In another study, Peng et al. found that combined transplantation of VEGF- and BMP-4-expressing muscle-derived stem cells lead to a more optimal bone formation compared with transplantation of BMP4-expressing cells alone [71]. Some studies have also revealed enhanced efficiency of new bone formation on a model of tibial fracture in sheep and rat models of femurs defects after the transplantation of AD-MSCs co-transfected with both BMP-2 and BMP-7 compared with those with cells expressing only BMP-2 or BMP-7 [64,65]. Furthermore, there is evidence that co-transfection of MSCs with microRNA (miR)-148b enhances BMP2-induced osteogenesis [68], and MSCs engineered with BMP-7- and IL-4 cDNA accelerates bone repair in mice [81].

CXC chemokine receptors (CXCRs) belong to a family of transmembrane proteins that specifically bind to CXC chemokines. At the moment, there are seven known CXCRs, and CXCR4 presented on the surface of MSCs is considered to be a key mediator of MSCs' rolling and engraftment [103-106]. It has been demonstrated that transduction of MSCs with adenovirus carrying CXCR4 and Cbfa 1 increased homing of MSCs to the defect site [83]. Using this rationale, Cho et al. prevented bone loss in ovariectomized (OVX) mice by intravenous transplantation of CXCR4-transfected MSCs [84]. Monocyte chemotactic protein, MIP-1 $\alpha$ and RANTEs are also involved in the process of MSCs homing, but to a lesser extent [107].

Cho et al. also demonstrated that bone loss in OVX mice could be reversed by MSCs overexpressing receptor activator of NF- $\mathrm{KB}$ (RANK-Fc) [84], and this finding was confirmed by Kim et al. [85]. RANK-Fc is a recombinant protein of RANK which acts as a soluble antagonist of RANKL to prevent osteoclastogenesis [108]. In a similar study, Akbar et al. demonstrated the indirect influence of AD-derived MSCs expressing Alpha-1 Antitrypsin on bone repair in OVX mice [86]. Alpha-1 Antitrypsin is a proteinase inhibitor which suppresses the release of proinflammatory cytokines, enhances the production of anti-inflammatory cytokine and reduces osteoclastassociated bone resorption [109].

Sox11 is a transcription factor which plays an important role in differentiation and migration of MSCs via activation of Runx2 and CXCR4 expression [87,110]. Recently, Xu et al. demonstrated that genetically modified MSCs displaying high expression of Sox 11 enhanced the differentiation and migration of MSCs and improved bone fracture healing in an open fracture model on Sprague-Dawley rats [87]. 
Runx2 has been identified as one of the transcription factors involved in osteoblast differentiation of MSCs [94]. Recent results showed that treatment of rat femurs bone defects with MSC genetically modified with Runx2 in spheroid cell implants accelerated bone repair as compared with nontransfected MSC spheroids [88]. Similarly, Deng and coauthors showed that knockdown of histone demethylase Jumonji AT-rich interactive domain 1A (Jarid1a) in bone MSCs of rats led to activation of Runx2 and subsequent improvement of calvarial bone defect regeneration [89].

As mentioned above, there are viral and nonviral vectors for the generation of genetically modified MSCs. Nonviral gene transfer for MSCs may generally be categorized in two groups: physical methods and chemical methods. Physical methods include gene guns, electroporation and sonoporation [73,74,111]. Another sufficiently effective method of a physical gene transfer is a nucleofection which is a variant of electroporation. The effectiveness of this method has been demonstrated in the studies where nucleofection of BMP- 6 gene was capable of enhancing osteogenic differentiation of AD-MSCs and BM-MSCs in vitro and in vivo [73,74]. Aslan et al. have reported that transplantation of hMSCs transfected with human BMP-2 or BMP-9 genes via nucleofection induced ectopic bone formation in NOD/SCID mice [80]. Chemical gene transfer approach involves application of the different nonviral carriers such as cationic lipids (liposome-based transfection) polymer-based systems and others [63,112,113]. In a comparison study, Park et al. evaluated liposome-mediated and adenoviral BMP-2 gene transfer of BM-MSCs in order to regenerate critical-size bone defects in rats [112]. The authors have established that liposome-mediated gene delivery into BM-MSCs was able to enhance bone repair albeit it took longer period than the adenoviral transfection.

The use of viral vectors has shown to be very effective in inducing the desired biological modifications in MSCs. This is in great part due to the very high level of transgene expression achieved by viral vectors. Among them, lentiviral vectors have the unique feature of allowing sustained transgene expression even after MSCs differentiation [114], which is often sought. Although integrating viral vectors such as adenovirus-associated virus, retrovirus and lentiviral vectors lead to persistent transgene expression, they also pose the potential risk of insertional mutagenesis, particularly retrovirus [115]. While the risk appears to be relatively small based on the information available today, it should still be taken into account when designing novel therapeutic strategies.

Notwithstanding the proven advantageous properties of MSCs in homing to sites of injury, inflammation and regeneration, there is evidence suggesting that MSCs may also promote tumor angiogenesis and development. Galderisi et al. indicate that tumors may be seen by MSCs as injury or regeneration sites, and as such may help create a microenvironment that promotes angiogenesis and metastasis of tumor cells [116]. Therefore, a word of caution in carefully selecting the applications of MSCs is also recommended. Even though the effect of MSCs on tumor progression has not been satisfactorily elucidated and can be considered debatable, the evidence of MSCs recruitment to tumors is quite strong [23]. Furthermore, the effect of MSCs on cancer stem cells demands important consideration and not fully established. Nevertheless, it is reasonable to assume that the context (such as the type of cancer cell line used in the study or the cytokines' profile) plays a key role in determining the ultimate effect of MSCs in promoting or inhibiting tumors. In this regard, simple activation or genetic engineering of MSCs may alter the context of the relationship between MSCs and tumor microenvironment leading to unforeseen consequences.

A further consideration is that for certain applications the expression of the therapeutic gene is only required for a short period of time, after which it is no longer required, and at times even unwanted. For instance, the time required for cytokines to exert its biological activity is quite short. Therefore, long-term expression of cytokines is often neither required nor recommended. Similarly, the cocktail of genes required to differentiate MSCs into the osteoblastic lineage is only required during the short period of time that it takes for the expressed transgene to induce changes in the cellular gene expression pattern. Once the differentiation process is underway the expression of the transgenes is no longer needed. Therefore, for certain applications, it is preferable to modify genetically MSCs transiently rather than permanently. In this regard, the use of episomal nonviral vectors potentially increases the safety of the treatment, although it is at the expense of lower transgene expression [117,118].

Additionally, newer transient cell engineering strategies based not on DNA but on the delivery of mRNA are available and have proven effective [119]. Mice transplanted with MSCs transiently transfected with mRNA for IL-10 and for homing ligands induced the homing to the cells to site of inflammation and reduced the inflammation without the need for persistent IL-10 expression [119]. MiRs are another key molecules that regulate the processes of cell differentiation [120], and there are several in vitro and in vivo studies indicating that miRs are capable of inducing osteogenic differentiation of human and rat AD-MSCs [68,91,120]. 
Table 3. Target moiety and corresponding agents for surface modifications of cells.

\begin{tabular}{|llr|}
\hline Target moiety & Agent & Ref. \\
\hline Hydroxyapatites & Bisphosphonates & [122,123] \\
\hline CXC4R & SDF-1 & [124] \\
\hline E-selectins & CD44 glycoform & {$[125]$} \\
\hline P-selectins & SLeX & {$[126]$} \\
\hline SDF-1: Stromal cell-derived factor-1; SLeX: Sialyl Lewis X. & & \\
\hline
\end{tabular}

\section{Surface modifications of MSCs}

According to Wu et al. at least 19 receptors are expressed on the surface of MSCs and all of them can potentially be used for cell targeting and homing [121]. These naturally occurring receptors may be effective in direct transplantation of MSCs without prior expansion in vitro. However, during in vitro proliferation of MSCs most of the receptors are found to be absent on the surface of culture-expanded cells [103]. This creates a whole niche for receptor or ligand engineering for targeted delivery of MSCs (Table 3).

For example, a receptor of particular interest, CX4CR, promotes MSCc rolling and binding to SDF- 1 that is present in bone marrow and ischemic tissue [104,105]. In this regards, Jones et al. primed MSCs with SDF-1 for $1 \mathrm{~h}$ before transplantation to increase the transcription expression level of CXCR4 receptor, and obtained an increased cell engraftment rate both in wild-type and in osteogenesis imperfecta mice, as well as improved bone quality and plasticity in response to fracture, especially in osteogenesis imperfecta mice animals [124].

As previously mentioned, MSCs have high affinity to inflamed tissues and there are data indicating that MSCs are actively recruited to the sites of inflammation via endothelial expressed P- and E- selectins [127]. The selectins belong to a family of Type 1 transmembrane cell adhesion molecules that mediate the initial step of leukocyte recruitment in the inflammatory process. The physiological ligands for selectins are numerous glycoproteins, including P-selectin glycoprotein ligand 1, E-selectin ligand 1, CD34 and CD44, and CD44 is present on the MSCs [127]. Sackstein et al. used a glycan engineering approach to enhance MSCs trafficking (targeting?) to bone. MSCs were modified ex vivo to change native CD44 glycoform on the surface of MSCs into hematopoietic cell E-selectin/L-selectin ligand. The results showed that MSCs accumulated in bone marrow within hours after systemic infusion [125]. Similarly, Sarkar $e t$ al. proposed the use of a nanometer-scale polymer construct containing sialyl Lewis X, also known as cluster of differentiation 15s (CD15s) or stage-specific embryonic antigen 1 to target MSCs to bone marrow. This molecule is a tetrasaccharide carbohydrate which is usually presented on the surface of leukocytes as an active binding site of selectins' ligands $[128,129]$ and promotes rolling and engraftment into the inflamed tissue with high expression of P-selectins [126].

In another study, researchers developed a two-end construct that binds to cell surface via a synthetic peptidomimetic ligand coupled to bisphosphonate (alendronate, Ale) [122]. The complex induced MSCs migration and differentiation along the osteogenic lineage in vitro. Intravenous injection of modified cells showed increased bone formation (especially trabecular) in estrogen deficient mice (role model of osteoporosis) by improving homing and retention of MSCs in bone tissue. The same polymer construct was also used in the study of D'Souza et al. [123]. Hydroxyl succinyl group was used for cell surface binding and alendronate was applied as a bone seeking agent. The polymer was synthesized using novel atom transfer radical polymerization (ATRP) technique to allow controlled polymerization of functional chains. In vitro data showed enhanced bone affinity compared with the polymer without bisphosphonate group. The polymer was not found to be toxic and therefore, opens the opportunity for its further testing in vivo.

\section{Conclusion}

MSCs cell therapy is undoubtedly becoming a reality for the treatment of bone-related conditions and fracture healing. Different approaches for cell transplantation and successful engraftment are being exploited to improve the healing outcomes. In this regard, cell engineering for bone regeneration attracts a lot of scientific attention making it of great clinical interest. There are three main strategies to increase the efficacy of MSCs transplantation: effective scaffold and hydrogel carriers, genetic modifications of MSCs and cell surface membrane engineering. Biomaterial scaffolds place the cells directly into the injury site; the genetic modifications of MSCs are aimed at improving the degree of engraftment via overexpression of genes controlling MSCs homing, proliferation and differentiation, whereas, the chemical engineering of the cell surface membrane provide bone targeting moieties to 
MSCs. Transplanting scaffolds populated with MSCs is a very suitable strategy for filling critical size bone defects, while transplantation of genetically or chemically modified MSCs would manage the inflammatory conditions that do not require scaffolds stability and can result in significantly enhanced bone regeneration in pathological fractures. All of the approaches discussed here hold a great potential for present and future clinical practices related to bone regeneration.

\section{Future perspective}

MSCs applications for bone regeneration have been extensively used in numerous preclinical and clinical studies. Despite the increasing interest of MSC-based regenerative therapies for bones and its promising clinical potentials, the limited therapeutic effects of MSC treatment remain a major challenge suggesting that the modifications of MSC are required. A comprehensive review of the available literature indicates that most efforts are focused on scaffolds development and genetic modifications of MSCs, while the number of studies aimed at nongenetic chemical cell surface modifications are limited. Although, the use of genetic modifications has shown to be very effective in conferring the desired biological properties to MSCs, they also pose potential mutagenesis and carcinogenesis risks and more studies are required to address biosafety issues. In contrast to genetic modifications that are mostly used to manipulate nucleic acids, cell surface engineering may be used to manipulate lipids, proteins or glycans on plasma membrane, potentially increasing the safety of the treatment. In our view, cell surface engineering that involves multidisciplinary approach and convergence of chemists and cell biologists is a very promising research direction that may lead to the development of safe and clinically relevant technologies of MSCs' based cell therapy. In any event, there is a need to further understand the biology of MSCs in the context of chemical/genetic modifications and its behavior upon transplantation.

\section{Executive summary}

- In pathological bone fracture conditions natural processes of bone regeneration are hindered, and, in this case, transplantation of mesenchymal stem cells (MSCs) is a promising therapeutic method to facilitate the healing processes.

- Managing homing affinity and improvement of cell viability, proliferation and differentiation are the primary tasks in MSC transplantation for bone regeneration.

Scaffolds \& hydrogels

- Seeding scaffolds with MSCs is a promising approach to increase the efficacy of scaffold transplants.

- One of the very important issues in cell-scaffold constructs is the lack of oxygen supply and nutrients.

- A number of recent studies have been directed toward creating bioactive scaffolds and hydrogels that increase the amount of oxygen available to MSCs, promote angiogenesis and support cell proliferation and differentiation.

Genetic modifications of MSCs

- Molecular targeting strategies for genetic modifications of MSCs are mainly focused on the activation of cytokines' secretion to promote MSC differentiation, increasing expression of the cell surface receptors responsible for cell rolling and migration and activation of transcription factors and other proteins responsible for the differentiation of MSCs.

- The use of viral vectors can give more stable results in terms of cell viability, proliferation and homing, though encounters major safety issues.

- The use of nonviral transfection potentially increases the safety of the treatment, although it is at the expense of lower transgene expression.

Surface modifications of MSCs

- During in vitro proliferation of MSCs, most of the receptors are found to be absent on the surface of culture-expanded cells and this creates a whole niche for receptor or ligand engineering for targeted delivery of MSCs.

- Surface binding of the different ligands to the cell membrane significantly reduces the potential risk of mutagenesis while allowing navigation of the cells precisely to the site of interest.

Conclusion \& future perspective

- All of the approaches discussed here hold a great potential for present and future clinical practices related to bone regeneration, yet there is a need to further understand the biology of MSCs in the context of chemical/genetic modifications and its behavior upon transplantation. 


\section{Author contributions}

Conceptualization: Sh Askarova and G Hortelano; funding acquisition: Sh Askarova; writing - original draft: Y Safarova, B Umbayev and Sh Askarova; writing - review \& editing: G Hortelano.

\section{Financial \& competing interests disclosure}

This work was supported by Grant of the Ministry of Education and Science of the Republic of Kazakhstan No. 0118 RK01040 and the CRP grant of Nazarbayev University No. 091019CRP2113. The authors have no other relevant affiliations or financial involvement with any organization or entity with a financial interest in or financial conflict with the subject matter or materials discussed in the manuscript apart from those disclosed.

No writing assistance was utilized in the production of this manuscript.

\section{Open access}

This work is licensed under the Attribution-NonCommercial-NoDerivatives 4.0 Unported License. To view a copy of this license, visit http://creativecommons.org/licenses/by-nc-nd/4.0/

\section{References}

Papers of special note have been highlighted as: • of interest;

1. Ensrud KE. Epidemiology of fracture risk with advancing age. J. Gerontol. A Biol. Sci. Med. Sci. 68(10), 1236-1242 (2013).

2. Pesce V, Speciale D, Sammarco G, Patella S, Spinarelli A, Patella V. Surgical approach to bone healing in osteoporosis. Clin. Cases Miner. Bone Metab. 6(2), 131-135 (2009).

3. Alonso N, Calero-Paniagua I, Del Pino-Montes J. Clinical and genetic advances in Paget's disease of bone: a review. Clin. Rev. Bone Miner. Metab. 15(1), 37-48 (2017).

4. Shaker JL. Paget's disease of bone: a review of epidemiology, pathophysiology and management. Ther. Adv. Musculoskelet. Dis. 1(2), 107-125 (2009).

5. Marcucci G, Brandi ML. Rare causes of osteoporosis. Clin. Cases Miner. Bone Metab. 12(2), 151-156 (2015).

6. Chan JK, Gotherstrom C. Prenatal transplantation of mesenchymal stem cells to treat osteogenesis imperfecta. Front. Pharmacol. 5,223 (2014).

7. Croes M, Van Der Wal BCH, Vogely HC. Impact of bacterial infections on osteogenesis: evidence from in vivo studies. J. Orthop. Res. 37(10), 2067-2076 (2019).

8. Stern SM, Ferguson PJ. Autoinflammatory bone diseases. Rheum. Dis. Clin. North Am. 39(4), 735-749 (2013).

9. Egusa H, Sonoyama W, Nishimura M, Atsuta I, Akiyama K. Stem cells in dentistry-Part II: clinical applications. J. Prosthodont. Res. 56(4), 229-248 (2012).

10. Klingemann H, Matzilevich D, Marchand J. Mesenchymal stem cells - sources and clinical applications. Transfus. Med. Hemother. 35(4), 272-277 (2008).

11. Panepucci RA, Siuf JL, Silva WA Jr. et al. Comparison of gene expression of umbilical cord vein and bone marrow-derived mesenchymal stem cells. Stem Cells 22(7), 1263-1278 (2004).

12. Strioga M, Viswanathan S, Darinskas A, Slaby O, Michalek J. Same or not the same? Comparison of adipose tissue-derived versus bone marrow-derived mesenchymal stem and stromal cells. Stem Cells Dev. 21(14), 2724-2752 (2012).

13. Ullah I, Subbarao RB, Rho GJ. Human mesenchymal stem cells - current trends and future prospective. Biosci. Rep. 35(2), e00191 (2015).

14. Kim Y, Kim H, Cho H, Bae Y, Suh K, Jung J. Direct comparison of human mesenchymal stem cells derived from adipose tissues and bone marrow in mediating neovascularization in response to vascular ischemia. Cell. Physiol. Biochem. 20(6), 867-876 (2007).

15. De Becker A, Riet IV. Homing and migration of mesenchymal stromal cells: how to improve the efficacy of cell therapy? World J. Stem Cells 8(3), 73-87 (2016).

16. Schrepfer S, Deuse T, Reichenspurner H, Fischbein MP, Robbins RC, Pelletier MP. Stem cell transplantation: the lung barrier. Transplant. Proc. 39(2), 573-576 (2007).

17. Fischer UM, Harting MT, Jimenez F et al. Pulmonary passage is a major obstacle for intravenous stem cell delivery: the pulmonary first-pass effect. Stem Cells Dev. 18(5), 683-692 (2009).

18. Nystedt J, Anderson H, Tikkanen J et al. Cell surface structures influence lung clearance rate of systemically infused mesenchymal stromal cells. Stem Cells 31(2), 317-326 (2013).

19. Eggenhofer E, Benseler V, Kroemer A et al. Mesenchymal stem cells are short-lived and do not migrate beyond the lungs after intravenous infusion. Front. Immunol. 3, 297 (2012). 
20. Gholamrezanezhad A, Mirpour S, Bagheri M et al. In vivo tracking of 111 In-oxine labeled mesenchymal stem cells following infusion in patients with advanced cirrhosis. Nucl. Med. Biol. 38(7), 961-967 (2011).

21. Ma S, Xie N, Li W, Yuan B, Shi Y, Wang Y. Immunobiology of mesenchymal stem cells. Cell Death Differ. 21(2), 216-225 (2014).

22. Sasaki M, Abe R, Fujita Y, Ando S, Inokuma D, Shimizu H. Mesenchymal stem cells are recruited into wounded skin and contribute to wound repair by transdifferentiation into multiple skin cell type. J. Immunol. 180(4), 2581-2587 (2008).

23. Kidd S, Spaeth E, Dembinski JL et al. Direct evidence of mesenchymal stem cell tropism for tumor and wounding microenvironments using in vivo bioluminescent imaging. Stem Cells 27(10), 2614-2623 (2009).

24. Cohn Yakubovich D, Sheyn D, Bez M et al. Systemic administration of mesenchymal stem cells combined with parathyroid hormone therapy synergistically regenerates multiple rib fractures. Stem Cell Res. Ther. 8(1), 51 (2017).

25. Sheyn D, Shapiro G, Tawackoli W et al. PTH induces systemically administered mesenchymal stem cells to migrate to and regenerate spine injuries. Mol. Ther. 24(2), 318-330 (2016).

26. Ghassemi T, Shahroodi A, Ebrahimzadeh MH, Mousavian A, Movaffagh J, Moradi A. Current concepts in scaffolding for bone tissue engineering. Arch. Bone Jt. Surg. 6(2), 90-99 (2018).

- Ghassemi et al. provided a comprehensive overview of all current concept of scaffold production.

27. Agacayak S, Gulsun B, Ucan MC, Karaoz E, Nergiz Y. Effects of mesenchymal stem cells in critical size bone defect. Eur. Rev. Med. Pharmacol. Sci. 16(5), 679-686 (2012).

28. Cancedda R, Mastrogiacomo M, Bianchi G, Derubeis A, Muraglia A, Quarto R. Bone marrow stromal cells and their use in regenerating bone. Novartis Found. Symp. 249 133-143 discussion 143-137, 170-134, 239-141 (2003).

29. Paduano F, Marrelli M, Amantea $\mathrm{M}$ et al. Adipose tissue as a strategic source of mesenchymal stem cells in bone regeneration: a topical review on the most promising craniomaxillofacial applications. Int. J. Mol. Sci. 18(10), E2140 (2017).

30. Sandor GK, Numminen J, Wolff J et al. Adipose stem cells used to reconstruct 13 cases with cranio-maxillofacial hard-tissue defects. Stem Cells Transl. Med. 3(4), 530-540 (2014).

31. Mesimaki K, Lindroos B, Tornwall J et al. Novel maxillary reconstruction with ectopic bone formation by GMP adipose stem cells. Int. J. Oral Maxillofac. Surg. 38(3), 201-209 (2009).

32. Wolff J, Sandor GK, Miettinen A et al. GMP-level adipose stem cells combined with computer-aided manufacturing to reconstruct mandibular ameloblastoma resection defects: experience with three cases. Ann. Maxillofac. Surg. 3(2), 114-125 (2013).

33. Sandor GK, Tuovinen VJ, Wolff J et al. Adipose stem cell tissue-engineered construct used to treat large anterior mandibular defect: a case report and review of the clinical application of good manufacturing practice-level adipose stem cells for bone regeneration. J. Oral Maxillofac. Surg. 71(5), 938-950 (2013).

34. Liu Y, Chan JK, Teoh SH. Review of vascularised bone tissue-engineering strategies with a focus on co-culture systems. J. Tissue Eng. Regen. Med. 9(2), 85-105 (2015).

35. Bai X, Gao M, Syed S, Zhuang J, Xu X, Zhang X-Q. Bioactive hydrogels for bone regeneration. Bioact. Mater. 3(4), $401-417$ (2018).

36. Kaigler D, Wang Z, Horger K, Mooney DJ, Krebsbach PH. VEGF scaffolds enhance angiogenesis and bone regeneration in irradiated osseous defects. J. Bone Miner. Res. 21(5), 735-744 (2006).

37. Bae JH, Song HR, Kim HJ et al. Discontinuous release of bone morphogenetic protein-2 loaded within interconnected pores of honeycomb-like polycaprolactone scaffold promotes bone healing in a large bone defect of rabbit ulna. Tissue Eng. Part A 17(19-20), 2389-2397 (2011).

38. Yu H, Vandevord PJ, Gong W et al. Promotion of osteogenesis in tissue-engineered bone by pre-seeding endothelial progenitor cells-derived endothelial cells. J. Orthop. Res. 26(8), 1147-1152 (2008).

39. Zhang W, Zhu C, Ye D et al. Porous silk scaffolds for delivery of growth factors and stem cells to enhance bone regeneration. PLoS ONE 9(7), e102371 (2014).

40. Kim SK, Cho TH, Han JJ, Kim IS, Park Y, Hwang SJ. Comparative study of BMP-2 alone and combined with VEGF carried by hydrogel for maxillary alveolar bone regeneration. Tissue Eng. Regen. Med. 13(2), 171-181 (2016).

41. Zhao X, Liu S, Yildirimer L et al. Injectable stem cell-laden photocrosslinkable microspheres fabricated using microfluidics for rapid generation of osteogenic tissue constructs. Adv. Funct. Mater. 26(17), 2809-2819 (2016).

42. He X, Dziak R, Mao K et al. Integration of a novel injectable nano calcium sulfate/alginate scaffold and BMP2 gene-modified mesenchymal stem cells for bone regeneration. Tissue Eng. Part A 19(3-4), 508-518 (2013).

43. Sayyar B, Dodd M, Marquez-Curtis L, Janowska-Wieczorek A, Hortelano G. Cell-matrix Interactions of Factor IX (FIX)-engineered human mesenchymal stromal cells encapsulated in RGD-alginate vs. Fibrinogen-alginate microcapsules. Artif. Cells Nanomed. Biotechnol. 42(2), 102-109 (2014).

44. Sayyar B, Dodd M, Wen J et al. Encapsulation of factor IX-engineered mesenchymal stem cells in fibrinogen-alginate microcapsules enhances their viability and transgene secretion. J. Tissue Eng. 3(1), 2041731412462018 (2012). 
45. Sayyar B, Dodd M, Marquez-Curtis L, Janowska-Wieczorek A, Hortelano G. Fibronectin-Alginate microcapsules improve cell viability and protein secretion of encapsulated Factor IX-engineered human mesenchymal stromal cells. Artif. Cells Nanomed. Biotechnol. 43(5), 318-327 (2015).

46. Huebsch N, Lippens E, Lee K et al. Matrix elasticity of void-forming hydrogels controls transplanted-stem-cell-mediated bone formation. Nat. Mater. 14(12), 1269-1277 (2015).

47. Paduano F, Marrelli M, White LJ, Shakesheff KM, Tatullo M. Odontogenic differentiation of human dental pulp stem cells on hydrogel scaffolds derived from decellularized bone extracellular matrix and collagen Type I. PLoS ONE 11(2), e0148225 (2016).

48. Kimelman-Bleich N, Pelled G, Sheyn D et al. The use of a synthetic oxygen carrier-enriched hydrogel to enhance mesenchymal stem cell-based bone formation in vivo. Biomaterials 30(27), 4639-4648 (2009).

- Interesting approach to use synthetic oxygen carrier to improve cell survival in hydrogel scaffold.

49. Benjamin S, Sheyn D, Ben-David S et al. Oxygenated environment enhances both stem cell survival and osteogenic differentiation. Tissue Eng. Part A 19(5-6), 748-758 (2013).

50. Papadimitropoulos A, Scotti C, Bourgine P, Scherberich A, Martin I. Engineered decellularized matrices to instruct bone regeneration processes. Bone 70, 66-72 (2015).

51. Wei W, Huang Y, Li D, Gou H-F, Wang W. Improved therapeutic potential of MSCs by genetic modification. Gene therapy 25, 538-547 (2018).

52. Jin YZ, Lee JH. Mesenchymal stem cell therapy for bone regeneration. Clin. Orthop. Surg. 10(3), 271-278 (2018).

53. Lu CH, Chang YH, Lin SY, Li KC, Hu YC. Recent progresses in gene delivery-based bone tissue engineering. Biotechnol. Adv. 31(8), 1695-1706 (2013).

54. Nowakowski A, Walczak P, Lukomska B, Janowski M. Genetic engineering of mesenchymal stem cells to induce their migration and survival. Stem Cells Int. 2016, 4956063-4956063 (2016).

55. Freitas J, Santos SG, Gonçalves RM, Teixeira JH, Barbosa MA, Almeida MI. Genetically engineered-MSC therapies for non-unions, delayed unions and critical-size bone defects. Int. J. Mol. Sci. 20(14), 3430 (2019).

56. Turgeman G, Pittman D, Müller R et al. Engineered human mesenchymal stem cells: a novel platform for skeletal cell mediated gene therapy. J. Gene Med. 3, 240-251 (2001).

57. Peterson B, Zhang J, Iglesias R et al. Healing of critically sized femoral defects, using genetically modified mesenchymal stem cells from human adipose tissue. Tissue Eng. 11(1-2), 120-129 (2005).

58. Miyazaki M, Zuk PA, Zou J, Yoon SH, Wei F, Morishita Y, Sintuu C, Wang JC et al. Comparison of human mesenchymal stem cells derived from adipose tissue and bone marrow for ex vivo gene therapy in rat spinal fusion model. Spine 33(8), 863-869 (2008)

59. Lieberman J, Daluiski A, Stevenson S et al. The effect of regional gene therapy with bone morphogenetic protein-2 producing bone marrow cells on the repair of segmental defects in rats. J. Bone Joint Surg. Am. 81, 905-917 (1999).

60. Kumar S, Nagy TR, Ponnazhagan S. Therapeutic potential of genetically modified adult stem cells for osteopenia. Gene Ther. 17(1), 105-116 (2010).

61. Pelled G, Tai K, Sheyn D et al. Structural and nanoindentation studies of stem cell-based tissue-engineered bone. J. Biomech. 40(2), 399-411 (2007).

62. Fernandes G, Wang C, Yuan X, Liu Z, Dziak R, Yang S. Combination of controlled release platelet-rich plasma alginate beads and bone morphogenetic protein-2 genetically modified mesenchymal stem cells for bone regeneration. J. Periodontol. 87(4), 470-480 (2016).

63. Tai K, Pelled G, Sheyn D et al. Nanobiomechanics of repair bone regenerated by genetically modified mesenchymal stem cells. Tissue Eng. Part A 14(10), 1709-1720 (2008).

64. Hernandez-Hurtado AA, Borrego-Soto G, Marino-Martinez IA et al. Implant composed of demineralized bone and mesenchymal stem cells genetically modified with AdBMP2/AdBMP7 for the regeneration of bone fractures in ovis aries. Stem Cells Int. 2016, 12 (2016).

65. Qing W, Guang-Xing C, Lin G, Liu Y. The osteogenic study of tissue engineering bone with BMP2 and BMP7 gene-modified rat adipose-derived stem cell. J. Biomed. Biotechnol. 2012, 7 (2012).

66. Samee M, Kasugai S, Kondo H, Ohya K, Shimokawa H, Kuroda S. Bone morphogenetic protein2 (BMP2) and vascular endothelial growth factor (VEGF) transfection to human periosteal cells enhances osteoblast differentiation and bone formation. J. Pharmacol. Sci. 108, 18-31 (2008).

67. Xiao C, Zhou H, Liu G et al. Bone marrow stromal cells with a combined expression of BMP-2 and VEGF-165 enhanced bone regeneration. Biomed. Mater. 6(1), 015013 (2011).

68. Liao Y-H, Chang Y-H, Sung L-Y et al. Osteogenic differentiation of adipose-derived stem cells and calvarial defect repair using baculovirus-mediated co-expression of BMP-2 and miR-148b. Biomaterials 35(18), 4901-4910 (2014).

69. Lin L, Fu X, Zhang X et al. Rat adipose-derived stromal cells expressing BMP4 induce ectopic bone formation in vitro and in vivo1. Acta Pharmacol. Sin. 27, 1608-1615 (2007).

70. Rose T, Peng H, Shen H-C et al. The role of cell type in bone healing mediated by ex vivo gene therapy. Langenbecks Arch. Surg. 388, 347-355 (2003). 
71. Peng H, Wright V, Usas A et al. Synergistic enhancement of bone formation and healing by stem cell-expressed VEGF and bone morphogenetic protein-4. J. Clin. Invest. 110(6), 751-759 (2002).

72. Sheyn D, Kallai I, Tawackoli W et al. Gene-modified adult stem cells regenerate vertebral bone defect in a rat model. Mol. Pharm. 8(5), 1592-1601 (2011).

73. Mizrahi O, Sheyn D, Tawackoli W et al. BMP-6 is more efficient in bone formation than BMP-2 when overexpressed in mesenchymal stem cells. Gene Ther. 20(4), 370-377 (2013).

74. Pelled G, Sheyn D, Tawackoli W et al. BMP6-engineered MSCs induce vertebral bone repair in a pig model: a pilot study. Stem Cells Int. 2016, 6530624-6530624 (2016).

- Shows that delivery of bone morphogenetic protein 6-modified mesenchymal stem cells (MSCs) can enhance bone formation in a in a clinically relevant, large animal pig model.

75. Liao H, Zhong Z, Liu Z, Li L, Ling Z, Zou X. Bone mesenchymal stem cells co-expressing VEGF and BMP-6 genes to combat avascular necrosis of the femoral head. Exp. Ther. Med. 15(1), 954-962 (2018).

76. Yan X, Zhou Z, Guo L et al. BMP7-overexpressing bone marrow-derived mesenchymal stem cells (BMSCs) are more effective than wild-type BMSCs in healing fractures. Exp. Ther. Med. 16(2), 1381-1388 (2018).

77. Dayoub H, Dumont RJ, Li JZ et al. Human mesenchymal stem cells transduced with recombinant bone morphogenetic protein-9 adenovirus promote osteogenesis in rodents. Tissue Eng. 9(2), 347-356 (2003).

78. Dumont R, Dayoub H, Li J et al. Ex Vivo bone morphogenetic protein-9 gene therapy using human mesenchymal stem cells induces spinal fusion in rodents. Neurosurgery 51 1239-1244 discussion 1244 (2002).

79. Wang X, Huang J, Huang F et al. Bone morphogenetic protein 9 stimulates callus formation in osteoporotic rats during fracture healing. Mol. Med. Rep. 15(5), 2537-2545 (2017).

80. Aslan H, Zilberman Y, Arbeli $\mathrm{V}$ et al. Nucleofection-based ex vivo nonviral gene delivery to human stem cells as a platform for tissue regeneration. Tissue Eng. 12(4), 877-889 (2006).

81. Lin T, Pajarinen J, Kohno Y et al. Transplanted interleukin-4-secreting mesenchymal stromal cells show extended survival and increased bone mineral density in the murine femur. Cytotherapy 20(8), 1028-1036 (2018).

82. Zhang H, Kot A, E Lay YA et al. Acceleration of fracture healing by overexpression of basic fibroblast growth factor in the mesenchymal stromal cells. Stem Cells Translational Medicine 6(10), 1880-1893 (2017).

83. Lien C-Y, Chih-Yuan Ho K, Lee OK, Blunn GW, Su Y. Restoration of bone mass and strength in glucocorticoid-treated mice by systemic transplantation of CXCR4 and Cbfa-1 co-expressing mesenchymal stem cells. J. Bone Miner. Res. 24(5), 837-848 (2009).

84. Cho SW, Sun HJ, Yang J-Y et al. Transplantation of mesenchymal stem cells overexpressing RANK-Fc or CXCR4 prevents bone loss in ovariectomized mice. Mol. Ther. 17(11), 1979-1987 (2009).

85. Kim D, Cho SW, Her SJ et al. Retrovirus-mediated gene transfer of receptor activator of nuclear factor- $\mathrm{KB}-\mathrm{Fc}$ prevents bone loss in ovariectomized mice. Stem Cells 24(7), 1798-1805 (2006).

86. Akbar MA, Lu Y, Elshikha AS et al. Transplantation of adipose tissue-derived mesenchymal stem cell (ATMSC) expressing alpha-1 antitrypsin reduces bone loss in ovariectomized osteoporosis mice. Hum. Gene Ther. 28(2), 179-189 (2016).

87. Xu L, Huang S, Hou Y et al. Sox11-modified mesenchymal stem cells (MSCs) accelerate bone fracture healing: sox11 regulates differentiation and migration of MSCs. FASEB J. 29(4), 1143-1152 (2014).

88. Yanagihara K, Uchida S, Ohba S, Kataoka K, Itaka K. Treatment of bone defects by transplantation of genetically modified mesenchymal stem cell spheroids. Mol. Ther. Meth. Clin. Dev. 9, 358-366 (2018).

- Demonstrates of osteogenic potential of transplanting genetically modified MSC spheroids with the Runx2 gene.

89. Deng Y, Guo T, Li J, Guo L, Gu P, Fan X. Repair of calvarial bone defect using Jarid1a-knockdown bone mesenchymal stem cells in rats. Tissue Eng Part A 24(9-10), 711-718 (2018).

90. Xie Q, Wang Z, Zhou H et al. The role of miR-135-modified adipose-derived mesenchymal stem cells in bone regeneration. Biomaterials 75, 279-294 (2016).

91. Deng Y, Zhou H, Zou D et al. The role of miR-31-modified adipose tissue-derived stem cells in repairing rat critical-sized calvarial defects. Biomaterials 34(28), 6717-6728 (2013).

92. Salazar VS, Gamer LW, Rosen V. BMP signalling in skeletal development, disease and repair. Nat. Rev. Endocrinol. 12(4), 203-221 (2016).

93. Wang RN, Green J, Wang Z et al. Bone morphogenetic protein (BMP) signaling in development and human diseases. Genes Dis. 1(1), 87-105 (2014).

94. Kang Q, Song W-X, Luo Q et al. A comprehensive analysis of the dual roles of BMPs in regulating adipogenic and osteogenic differentiation of mesenchymal progenitor cells. Stem Cells Dev. 18(4), 545-559 (2009).

95. El Bialy I, Jiskoot W, Reza Nejadnik M. Formulation, delivery and stability of bone morphogenetic proteins for effective bone regeneration. Pharm. Res. 34(6), 1152-1170 (2017). 
96. Musgrave DS, Bosch P, Ghivizzani S, Robbins PD, Evans CH, Huard J. Adenovirus-mediated direct gene therapy with bone morphogenetic protein-2 produces bone. Bone 24(6), 541-547 (1999).

97. Bougioukli S, Sugiyama O, Pannell W et al. Gene therapy for bone repair using human cells: superior osteogenic potential of bone morphogenetic protein 2-transduced mesenchymal stem cells derived from adipose tissue compared to bone marrow. Hum. Gene Ther. 29(4), 507-519 (2018).

- Indicates that adipose tissue might be a preferable source of MSCs to develop a regional gene therapy approach to treat difficult bone-repair scenarios.

98. Tsuji K, Cox K, Bandyopadhyay A, Harfe BD, Tabin CJ, Rosen V. BMP4 is dispensable for skeletogenesis and fracture-healing in the limb. J. Bone Joint Surg. Am. 90(Suppl. 1), 14-18 (2008).

99. Rose T, Peng H, Shen H-C et al. The role of cell type in bone healing mediated by ex vivo gene therapy. Langenbecks Arch. Surg. 388(5), 347-355 (2003).

100. Cecchi S, Bennet SJ, Arora M. Bone morphogenetic protein-7: review of signalling and efficacy in fracture healing. J. Orthop. Transl. 4, 28-34 (2016).

101. Cui J, Zhang W, Huang E et al. BMP9-induced osteoblastic differentiation requires functional Notch signaling in mesenchymal stem cells. Lab. Invest. 99(1), 58-71 (2019).

102. Li B, Wang H, Qiu G, Su X, Wu Z. Synergistic effects of vascular endothelial growth factor on bone morphogenetic proteins induced bone formation in vivo: influencing factors and future research directions. Biomed. Res. Int. 2016, 2869572-2869572 (2016).

103. Karp JM, Leng Teo GS. Mesenchymal stem cell homing: the devil is in the details. Cell Stem Cell 4(3), 206-216 (2009).

104. Shi M, Li J, Liao L et al. Regulation of CXCR4 expression in human mesenchymal stem cells by cytokine treatment: role in homing efficiency in NOD/SCID mice. Haematologica 92(7), 897-904 (2007).

105. Potapova IA, Brink PR, Cohen IS, Doronin SV. Culturing of human mesenchymal stem cells as three-dimensional aggregates induces functional expression of CXCR4 that regulates adhesion to endothelial cells. J. Biol. Chem. 283(19), 13100-13107 (2008).

106. Su P, Tian Y, Yang C et al. Mesenchymal stem cell migration during bone formation and bone diseases therapy. Int. J. Mol. Sci. 19(8), (2018).

107. Ito H. Chemokines in mesenchymal stem cell therapy for bone repair: a novel concept of recruiting mesenchymal stem cells and the possible cell sources. Mod. Rheumatol. 21(2), 113-121 (2011).

108. Hsu H, Lacey DL, Dunstan CR et al. Tumor necrosis factor receptor family member RANK mediates osteoclast differentiation and activation induced by osteoprotegerin ligand. Proc. Natl Acad. Sci. USA 96(7), 3540-3545 (1999).

109. Janciauskiene S, Larsson S, Larsson P, Virtala R, Jansson L, Stevens T. Inhibition of lipopolysaccharide-mediated human monocyte activation, in vitro, by a1-antitrypsin. Biochem Biophys Res Commun 321(3), 592-600 (2004).

110. Larson BL, Ylostalo J, Lee RH, Gregory C, Prockop DJ. Sox11 is expressed in early progenitor human multipotent stromal cells and decreases with extensive expansion of the cells. Tissue Eng. Part A 16(11), 3385-3394 (2010).

111. Li W, Wei H, Xia C et al. Gene gun transferring-bone morphogenetic protein 2 (BMP-2) gene enhanced bone fracture healing in rabbits. Int. J. Clin. Exp. Med. 8, 19982-19993 (2016).

112. Park J, Ries J, Gelse K et al. Bone regeneration in critical size defects by cell-mediated BMP-2 gene transfer: a comparison of adenoviral vectors and liposomes. Gene Ther. 10(13), 1089-1098 (2003).

113. Raftery RM, Mencía-Castaño I, Sperger $S$ et al. Delivery of the improved BMP-2-Advanced plasmid DNA within a gene-activated scaffold accelerates mesenchymal stem cell osteogenesis and critical size defect repair. J. Control. Release 283, 20-31 (2018).

114. Zhang XY, La Russa VF, Bao L, Kolls J, Schwarzenberger P, Reiser J. Lentiviral vectors for sustained transgene expression in human bone marrow-derived stromal cells. Mol. Ther. 5(5 Pt 1), 555-565 (2002).

115. Hacein-Bey-Abina S, Von Kalle C, Schmidt M et al. LMO2-associated clonal T cell proliferation in two patients after gene therapy for SCID-X1. Science 302(5644), 415-419 (2003).

116. Galderisi U, Giordano A, Paggi MG. The bad and the good of mesenchymal stem cells in cancer: boosters of tumor growth and vehicles for targeted delivery of anticancer agents. World J. Stem Cells 2(1), 5-12 (2010).

117. Yin H, Kanasty RL, Eltoukhy AA, Vegas AJ, Dorkin JR, Anderson DG. Non-viral vectors for gene-based therapy. Nat. Rev. Genet. 15(8), 541-555 (2014).

118. Hamann A, Nguyen A, Pannier AK. Nucleic acid delivery to mesenchymal stem cells: a review of nonviral methods and applications. $J$. Biol. Eng. 13, 7-7 (2019).

119. Levy O, Zhao W, Mortensen LJ et al. mRNA-engineered mesenchymal stem cells for targeted delivery of interleukin-10 to sites of inflammation. Blood 122(14), e23-32 (2013).

120. Bartel DP. MicroRNAs: target recognition and regulatory functions. Cell 136(2), 215-233 (2009).

121. Wu Y, Zhao RC. The role of chemokines in mesenchymal stem cell homing to myocardium. Stem Cell Rev. 8(1), 243-250 (2012).

122. Guan M, Yao W, Liu R et al. Directing mesenchymal stem cells to bone to augment bone formation and increase bone mass. Nat. Med. 18(3), 456-462 (2012). 
- Shows the use of bisphopsphonate affinity for bone targeting polymer construct to navigate the MSCs.

123. D'souza S, Murata H, Jose MV et al. Engineering of cell membranes with a bisphosphonate-containing polymer using ATRP synthesis for bone targeting. Biomaterials 35(35), 9447-9458 (2014).

- Proposes the novel method of bisphosphonate polymer synthesis using ATRP for navigation of MSCs to the bone surface.

124. Jones GN, Moschidou D, Lay K et al. Upregulating CXCR4 in human fetal mesenchymal stem cells enhances engraftment and bone mechanics in a mouse model of osteogenesis imperfecta. Stem Cells Transl. Med. 1(1), 70-78 (2012).

125. Sackstein R. Directing stem cell trafficking via GPS. Methods Enzymol. 479, 93-105 (2010).

126. Sarkar D, Spencer JA, Phillips JA et al. Engineered cell homing. Blood 118(25), e184-e191 (2011).

- One of the pioneering ideas of cell surface modification for enhance targeting to the inflamed tissue.

127. Yilmaz G, Vital S, Yilmaz CE, Stokes KY, Alexander JS, Granger DN. Selectin-mediated recruitment of bone marrow stromal cells in the postischemic cerebral microvasculature. Stroke 42(3), 806-811 (2011).

128. Vestweber D, Blanks JE. Mechanisms that regulate the function of the selectins and their ligands. Physiol. Rev. 79(1), 181-213 (1999).

129. Zhang X, Bogorin DF, Moy VT. Molecular basis of the dynamic strength of the sialyl Lewis X-selectin interaction. Chemphyschem 5(2), 175-182 (2004). 